\title{
Morphologic and Molecular Characterization of a Demodex (Acari: Demodicidae) Species from White-Tailed Deer (Odocoileus virginianus)
}

\author{
Michael J. Yabsley, ${ }^{1,2}$ Sarah E. Clay, ${ }^{1}$ Samantha E. J. Gibbs, ${ }^{1,3}$ \\ Mark W. Cunningham, ${ }^{4}$ and Michaela G. Austel ${ }^{5,6}$ \\ ${ }^{1}$ Southeastern Cooperative Wildlife Disease Study, Department of Population Health, \\ The University of Georgia College of Veterinary Medicine, Wildlife Health Building, Athens, GA 30602, USA \\ ${ }^{2}$ Warnell School of Forestry and Natural Resources, The University of Georgia, Athens, GA 30602, USA \\ ${ }^{3}$ Division of Migratory Bird Management, U.S Fish \& Wildlife Service, Laurel, MD 20708, USA \\ ${ }^{4}$ Florida Fish and Wildlife Conservation Commission, Gainesville, FL 32653, USA \\ ${ }^{5}$ Department of Small Animal Medicine and Surgery, The University of Georgia College of Veterinary Medicine, University of Georgia, \\ Athens, GA 30602, USA \\ ${ }^{6}$ Massachusetts Veterinary Referral Hospital, Woburn, MA 01801, USA
}

Correspondence should be addressed to Michael J. Yabsley; myabsley@uga.edu

Received 26 October 2012; Accepted 15 November 2012

Academic Editors: G. Mkoji, P. Somboon, and J. Venegas Hermosilla

Copyright (C) 2013 Michael J. Yabsley et al. This is an open access article distributed under the Creative Commons Attribution License, which permits unrestricted use, distribution, and reproduction in any medium, provided the original work is properly cited.

\begin{abstract}
Demodex mites, although usually nonpathogenic, can cause a wide range of dermatological lesions ranging from mild skin irritation and alopecia to severe furunculosis. Recently, a case of demodicosis from a white-tailed deer (Odocoileus virginianus) revealed a Demodex species morphologically distinct from Demodex odocoilei. All life cycle stages were considerably larger than D. odocoilei and although similar in size to D. kutzeri and D. acutipes from European cervids, numerous morphometrics distinguished the four species. Adult males and females were $209.1 \pm 13.1$ and $225.5 \pm 13.4 \mu \mathrm{m}$ in length, respectively. Ova, larva, and nymphs measured $65.1 \pm 4.1,124.9 \pm 11.6$, and $205.1 \pm 19.4 \mu \mathrm{m}$ in length, respectively. For phylogenetic analyses, a portion of the $18 \mathrm{~S}$ rRNA gene was amplified and sequenced from samples of the WTD Demodex sp., two Demodex samples from domestic dogs, and Demodex ursi from a black bear. Phylogenetic analyses indicated that the WTD Demodex was most similar to D. musculi from laboratory mice. A partial sequence from D. ursi was identical to the WTD Demodex sequence; however, these two species can be differentiated morphologically. This paper describes a second Demodex species from white-tailed deer and indicates that $18 \mathrm{~S}$ rRNA is useful for phylogenetic analysis of most Demodex species, but two morphologically distinct species had identical partial sequences. Additional gene targets should be investigated for phylogenetic and parasite-host association studies.
\end{abstract}

\section{Introduction}

Mites of the genus Demodex are commonly found in the hair follicles and sebaceous glands of most mammals. In general, Demodex are considered to be host-species specific and some hosts can be infested with two or more distinct species (e.g., D. canis, D. injai, an undescribed short form Demodex sp. in dogs, $D$. brevis and D. folliculorum in humans, D. odocoilei in white-tailed deer (Odocoileus virginianus), and D. bovis in cattle) [1-5]. In general, Demodex infestations can vary widely in clinical presentation (from asymptomatic animals to cases with variable extends of alopecia, varying degrees of thickening of the skin, to cutaneous nodular lesions and severe dermatitis/furunculosis). In small animals, some animals have concurrent immunosuppression; however, it is unclear what role immunosuppression plays in generalized clinical demodicosis. In addition, some individuals or species, especially cervids in Europe, as well as cattle, develop 
a nodular demodicosis $[3,6]$. In a longitudinal study of cattle, these nodules waxed and waned both in number and size over time [6].

A Demodex sp. was first detected in a white-tailed deer from Georgia in the 1960s [7]. This infested deer exhibited hair loss and thickening of the skin on the head, neck, and shoulders. A second incidence of demodicosis was reported in Oklahoma in 1971 from a white-tailed deer with alopecia [8]. Demodex odocoilei, currently the only known Demodex sp. from white-tailed deer in the United States, was described using material from skin scrapings of deer in Georgia, Virginia, and Oklahoma [4]. These mites were obtained via deep skin scrapings of formalinfixed tissue. Histology of the skin samples did indicate hair loss and distention of the hair follicle and sebaceous glands, but the presence of mites was not associated with inflammation [4]. Mites morphologically consistent with D. odocoilei were reported from Columbian black-tailed deer (Odocoileus hemionus columbianus) and mule deer (Odocoileus hemionus hemionus) from the western United States and Canada [9, 10]. Recently, Demodex kutzeri, a species of European cervids, was reported from three species of cervids (one mule deer and one Rocky Mountain elk (Cervus elaphus nelsoni) from Colorado and a white-tailed deer from South Dakota) as well as a white-tailed deer from South Carolina (since 1971) [5]. Throughout the southeastern United States, numerous other cases of demodicosis have been diagnosed in white-tailed deers (SCWDS, unpublished data); however, the causative species of Demodex was not determined.

Demodex is currently classified in the subclass Acari, superorder Acariformes, order Prostigmata, superfamily Chelyetoidea, and family Demodicidae. Although PCR and sequence analysis have served as vital techniques to investigate relationships of numerous organisms, including mites, there is currently only genetic information available for four Demodex species, D. folliculorum and D. brevis from humans, D. canis from a dog [11], and D. musculi from mice in Genbank (accession number JF834894). The increased availability of sequence data would allow researchers to investigate the diversity of Demodex species infecting different or the same host species, host specificity, and investigate any geographic variability among Demodex (e.g., D. canis from various continents).

A sample of Demodex from a white-tailed deer clinical case submitted to the Southeastern Cooperative Wildlife Disease Study (SCWDS) was found to differ morphologically from $D$. odocoilei and other Demodex species reported from cervids. In this study, our goal was to characterize this Demodex sp. using a combination of morphological and molecular techniques. The morphologic characters of this Demodex sp. were compared to other cervid Demodex including D. odocoilei, D. acutipes, and D. kutzeri $[3,4,12]$. For our molecular analysis, we compared the Demodex sp. from the white-tailed deer with two Demodex samples from dogs, Demodex ursi from a black bear, and related sequences available in GenBank from Demodex and related mites.

\section{Materials and Methods}

In October 2002, a 2.5-year-old female hunter-killed deer from Lee County, South Carolina (USA) was submitted to SCWDS for diagnostic evaluation because of grossly visible skin lesions. A complete necropsy was performed and samples of skin were fixed in $10 \%$ buffered formalin, embedded in paraffin, sectioned, and stained with hematoxylin and eosin. Other portions of skin were preserved in $100 \%$ ethanol for PCR analysis.

Demodex mites removed from nodules were placed in immersion oil and covered with a cover slip. Mites were examined on 400X power and measurements of all life stages (adult male and female, nymph, larva, and ovum) were taken using a calibrated micrometer. Measurement of the adults included total body length, gnathosomal length, podosomal length, opisthosomal length, and presence of and length of aedeagus or vulva as appropriate. Measurements of ova, larvae, and nymphs included total length and width for ova. A total of 38 ova, 38 larvae, 40 nymphs, 42 female, and 44 male mites were measured. The Average and standard deviation for each measurement category were calculated.

Samples for molecular characterization were collected from various sources. Skin nodules from the white-tailed deer clinical case had been preserved in 100\% ethanol, skin samples of $D$. ursi from a black bear from Florida (USA) with generalized demodicosis were stored at $-20^{\circ} \mathrm{C}$ until analysis, and samples of $D$. canis and a short form Demodex sp. from dogs from Clarke County, Georgia (USA) were obtained as fresh skin scrapings. Each individual sample was placed into a Sarstedt O-ring tube with two copper BBs. The sample was macerated for 2 one-minute cycles at $1 / 2$-speed setting with a Mini Beadbeater 8 (Biospec Products, Bartlesville, OK). Phosphate-buffered saline $(200 \mu \mathrm{L})$ was added to each tube and the samples were vortexed thoroughly. DNA was extracted using the GFX Genomic Blood DNA Purification Kit (Amersham Biosciences, Piscataway, NJ, USA) according to the manufacturer's directions for direct blood. Because the primers were designed for ticks, we used three tick species as positive controls. DNA was extracted from three individual ticks (Dermacentor variabilis, Amblyomma americanum, and Haemaphysalis leporispalustris) as described for the mites except that they were individually frozen in liquid nitrogen before being macerated. Water controls were included in each step of the PCR analyses (extraction, primary reaction, and secondary reaction) to serve as negative controls.

Overlapping primer pairs that amplify the $18 \mathrm{~S}$ rRNA gene of the ticks and other mites were selected to provide near full-length sequence data for Demodex [13]. Primary reactions were conducted with primers NS1/NS8 and NS1/NS4. Overlapping nested and heminested secondary PCR reactions with primer pairs NS1/NS2, NS12+/NS2, NS1/NS4, and NS58.1/NS8 were conducted as described [13]. Briefly, each $25 \mu \mathrm{L}$ reaction contained $11 \mu \mathrm{L}$ molecular biology grade water, $2.5 \mu \mathrm{L} 25 \mathrm{mM} \mathrm{MgCl}_{2}, 5 \mu \mathrm{L} 5 \mathrm{X}$ colorless buffer (Promega, Madison, WI), $0.25 \mu \mathrm{L}$ of $20 \mathrm{mM}$ dNTPs (Promega), $0.5 \mu \mathrm{L}$ each primer $(50 \mu \mathrm{M})$, and $0.25 \mu \mathrm{L}$ GoTaq Flexi (Promega). Reaction conditions consisted of $1 \mathrm{~min}$ at $92^{\circ} \mathrm{C}$ followed by 10 cycles of $1 \mathrm{~min}$ at $92^{\circ} \mathrm{C}, 1$ minute at $48^{\circ} \mathrm{C}$, 
TABle 1: Measurements of ova, larvae, and nymphs of Demodex species from cervids.

\begin{tabular}{|c|c|c|c|}
\hline & Demodex sp. & D. kutzeri ${ }^{\mathrm{b}}$ & D. odocoilei ${ }^{\mathrm{c}}$ \\
\hline \multicolumn{4}{|l|}{ Ovum } \\
\hline Length $(\mu \mathrm{m} \pm \mathrm{SD})$ & $\begin{array}{l}65.1 \pm 4.1 \\
(n=38)^{\mathrm{a}}\end{array}$ & $74 \pm 6.2$ & $59.1 \pm 3.5$ \\
\hline Width & $38.7 \pm 3.8$ & $50 \pm 5.5$ & $26.9 \pm 3.7$ \\
\hline Larva length & $\begin{array}{c}124.9 \pm 11.6 \\
(n=38)\end{array}$ & $121.4 \pm 11.1$ & $93.5 \pm 11.2$ \\
\hline Nymph length & $\begin{array}{c}205.1 \pm 19.4 \\
(n=40)\end{array}$ & $253.7 \pm 38.8$ & $167.8 \pm 17.9$ \\
\hline
\end{tabular}

${ }^{a} n$ : the number of each stage measured.

${ }^{\mathrm{b}}$ See [3].

${ }^{\mathrm{c}}$ See [4].

and $90 \mathrm{sec}$ at $72^{\circ} \mathrm{C}$, then $32 \mathrm{cycles}$ of $1 \mathrm{~min}$ at $92^{\circ} \mathrm{C}, 35 \mathrm{sec}$ at $54^{\circ} \mathrm{C}$, and $90 \mathrm{sec}$ at $72^{\circ} \mathrm{C}$, and a final extension time of $7 \mathrm{~min}$ at $72^{\circ} \mathrm{C}$. Amplicons were visualized in a $2 \%$ agarose gel stained with ethidium bromide. Amplicons were purified using the QIAquick Gel Purification Kit (Qiagen, Valencia, CA, USA) and bidirectionally sequenced at the Integrated Biotechnology Laboratories (University of Georgia, Athens, GA, USA).

Sequences obtained from this study and from related organisms stored in GenBank were aligned using the multisequence alignment Clustal algorithm in MEGA version 3.1 [14]. GenBank accession numbers for the Demodex sp. from white-tailed deer, D. canis, the short form Demodex sp. from a dog, and D. ursi are KC010483, KC010485, KC010484, and KC010482, respectively. Phylogenetic analyses were conducted using MEGA version 3.1 program [14] using the minimum evolution algorithm with the Kimura 2-parameter model.

\section{Results}

3.1. Case Description. The deer weighed $58 \mathrm{~kg}$ and was in good physical condition with adequate fat deposits around the kidneys and within the mesentery. The only gross lesion noted during necropsy was the presence of multiple $\sim 1$ $2 \mathrm{~cm}$ tan cutaneous nodules on the head, legs, and lateral aspects of the thorax and abdomen, but no alopecia was noted (Figure 1). The nodules, when examined microscopically, were shown to contain several hundreds to thousands of intrafollicular Demodex mites (Figures 2 and 3). Histologically, hair follicles were extremely enlarged and the cysts were lined by stratified squamous epithelium varying between three and more than 20 cells in thickness (Figure 4). The majority of follicles were not surrounded by inflammatory cells (Figures 2, 3, and 4), but some cysts were disrupted resulting in infiltration with lymphocytes, plasma cells, and eosinophils (Figure 5). Skin samples also displayed a perifollicular lymphocytic to granulomatous dermatitis with scattered eosinophils. Marked fibrosis was noted around some affected hair follicles. Occasional giant granulomas with lymphocytes and scattered eosinophils were noted.

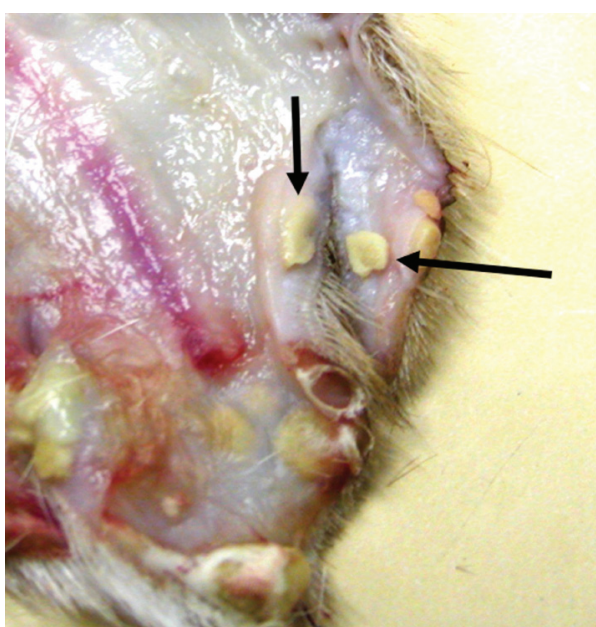

FIGURE 1: Gross presentation showing multifocal tan cutaneous nodules (arrows) containing numerous Demodex mites.

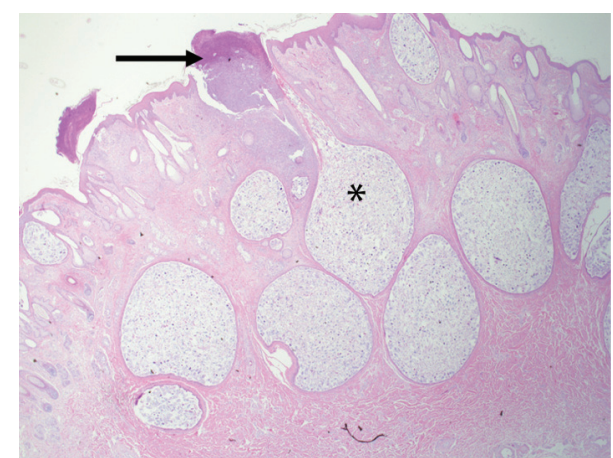

Figure 2: Multiple cystic structures filled with extremely high numbers of mites $\left(^{*}\right)$. High accumulation of eosinophils in the area of serocellular crusting (arrow).

3.2. Morphology. The Demodex sp. from the white-tailed deer in this study was most similar morphologically to $D$. kutzeri and $D$. acutipes from European red deer $[3,12]$ and is easily differentiated from $D$. odocoilei from whitetailed deer [4] (Figures 6 and 7). Numerous morphological measurements of larval, nymphal, and adult mites can be used to differentiate the Demodex sp. from $D$. odocoilei from whitetailed deer (Tables 1 and 2). This Demodex sp. can also be differentiated from $D$. acutipes and $D$. kutzeri of red deer by some measurements of adult mites (e.g., width of podosoma and opisthosoma and length of aedeagus) (Table 2).

3.3. Molecular Characterization. Overlapping sequences obtained from the Demodex sp. of WTD, Demodex canis, and the Demodex sp. (short form) from the dog were 1,035, 1,029 and $1,020 \mathrm{bp}$, respectively. The two canine Demodex sequences only differed by a single base and differed from the Demodex from the WTD by $34 \mathrm{bp}$. The $766 \mathrm{bp}$ obtained from D. ursi from the black bear was identical to the overlapping sequence of the Demodex sp. of WTD. The final alignment of the Demodex spp. from the WTD and dogs with related organisms (shown in Figure 8) was 1,093 bp; 405 bp was 


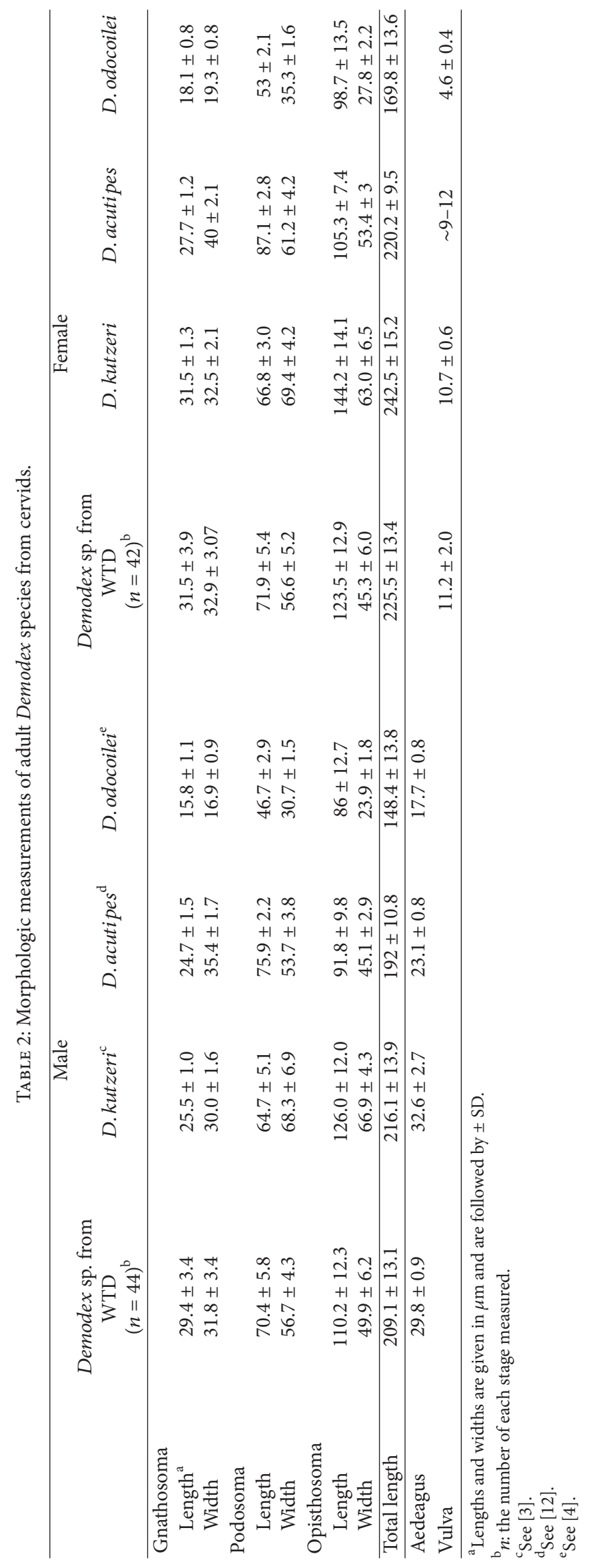




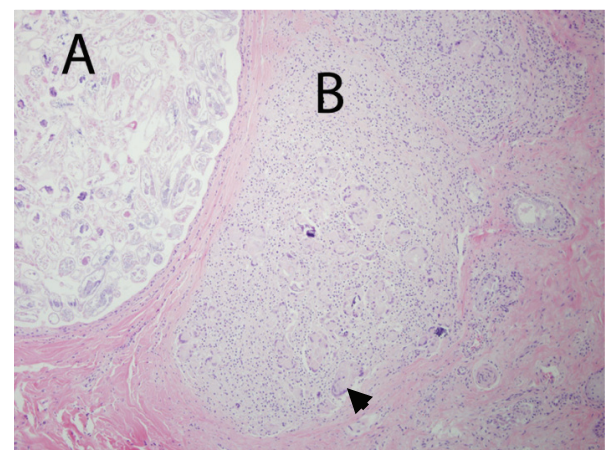

FIGURE 3: (A) Cystic structure filled with mites and no inflammation present. (B) Ruptured cyst with mite remnants and multinucleated giant cells (arrowhead).

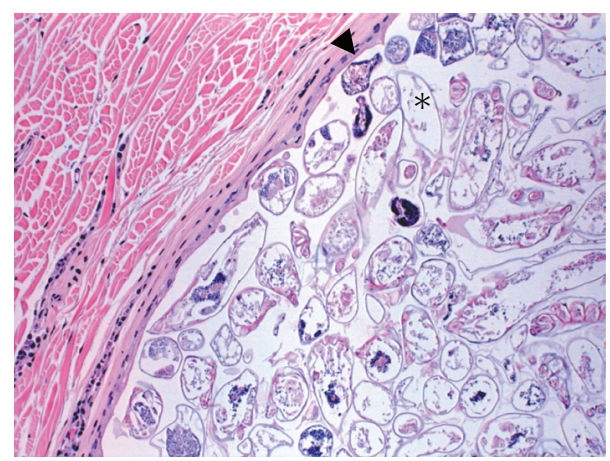

FIgURe 4: Wall of a single cyst filled with Demodex mites $\left(^{*}\right)$ showing the stratified squamous epithelium (arrowhead) and lack of inflammatory response around the cyst.

variable and of those 267 was parsimonious informative. The samples from the genus Demodex formed a monophyletic clade with $D$. brevis from humans at the base. The Demodex sp. of WTD formed a monophyletic clade with D. musculi from laboratory mice (JF834894) and these were in a clade with $D$. folliculorum from humans (Figure 8). This clade was a sister clade to various $D$. canis samples. Basal to the Demodex clade was Neochelacheles messersmithi, another mite in the superfamily Chelyetoidea, which inhabits spore

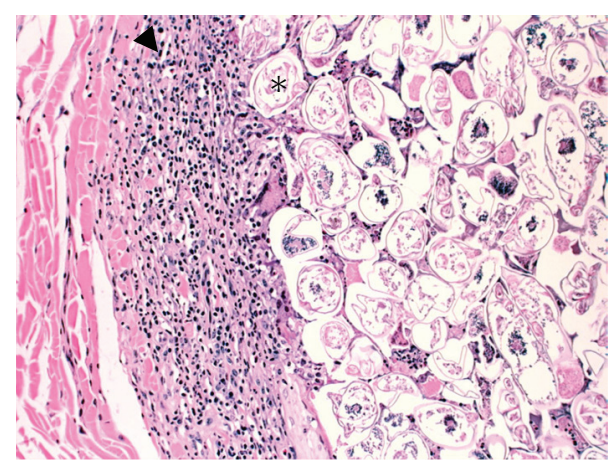

FIgURE 5: Disruption of this cyst filled with Demodex mites $\left({ }^{*}\right)$ which has resulted in an inflammatory infiltration (arrowhead).

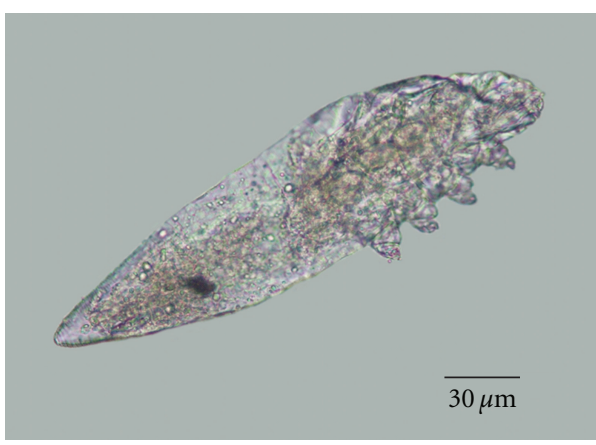

FIGURE 6: Oil mounted single mite obtained from the clinical case.

tubes of polypore fungi and preys on astigmatic mites [15]. The separation of Demodex spp. from N. messersmithi was well supported (bootstrap 100\%).

\section{Discussion and Conclusions}

Since 1977, 16 cases of demodicosis in white-tailed deer have been documented at SCWDS (unpublished data), and when combined with previously documented cases, Demodexinfested white-tailed deer have been detected throughout the host range (Maine, South Dakota, Texas, and numerous southeastern and Midwestern states) [4, 5, 7, 8, 16-18]. In only a few of these cases were the mites morphologically determined, and most were identified as D. odocoilei [4], but recently, three deer were infested with $D$. kutzeri [5]. Infection of individual mammalian species with multiple species of Demodex is common (e.g., three species in domestic dogs, two in domestic cats); thus the finding of a third Demodex species infesting white-tailed deer is not unexpected. The Demodex sp. identified in this clinical case was on average 30\% larger than Demodex odocoilei but was morphologically similar to D. acutipes and D. kutzeri, both parasites of red deer (Cervus elaphus) from Europe, and the latter was recently reported from three species of cervids from North America [5]. However, the Demodex sp. can be differentiated from both by several morphologic measurements.

Similar to the initial description of D. odocoilei [4], the Demodex sp. in this paper inhabits the hair follicles and sebaceous glands of the white-tailed deer. Similar to observations with $D$. odocoilei, little to no inflammation was associated with the majority of hair follicles and sebaceous glands containing the mites. However, in contrast to $D$. odocoilei-infected deer, the Demodex sp.-infected deer had hair follicles that were severely distended and contained thousands of mites within giant cystic, follicular structures. The gross presentation was more similar to that described for D. kutzeri and D. acutipes from red deer in Europe $[3,12]$ and D. kutzeri from three species of cervids from Colorado, South Dakota, and South Carolina [5]. The case material used in this study was obtained from a clinical case submission of a noncaptive hunter-killed deer; therefore, we were unable to investigate the chronicity of lesions associated with the Demodex sp. in white-tailed deer. Similar to previous reports of D. kutzeri in cervids, the current clinical case presented 


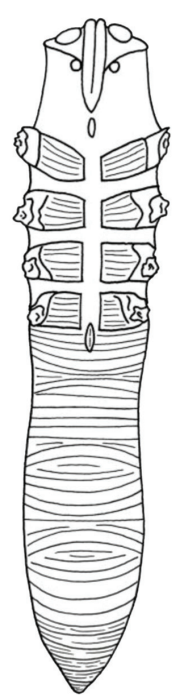

(a)

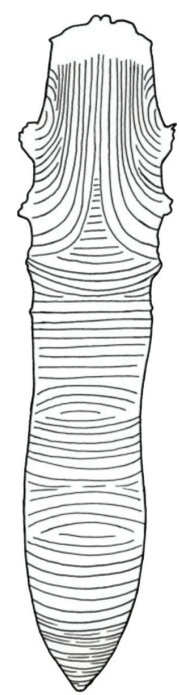

(b)

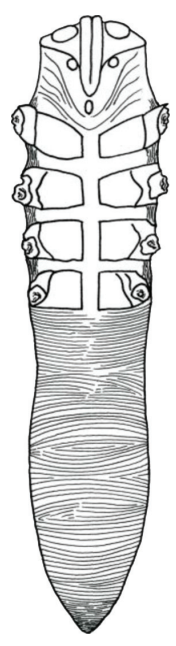

(c)

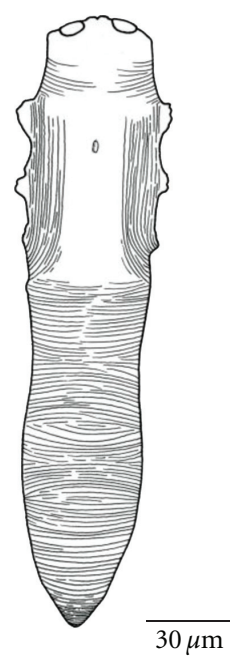

(d)

FIgURE 7: Line drawings of the Demodex sp. from white-tailed deer. Female ((a) ventral; (b) dorsal) and male ((c) ventral; (d) dorsal).

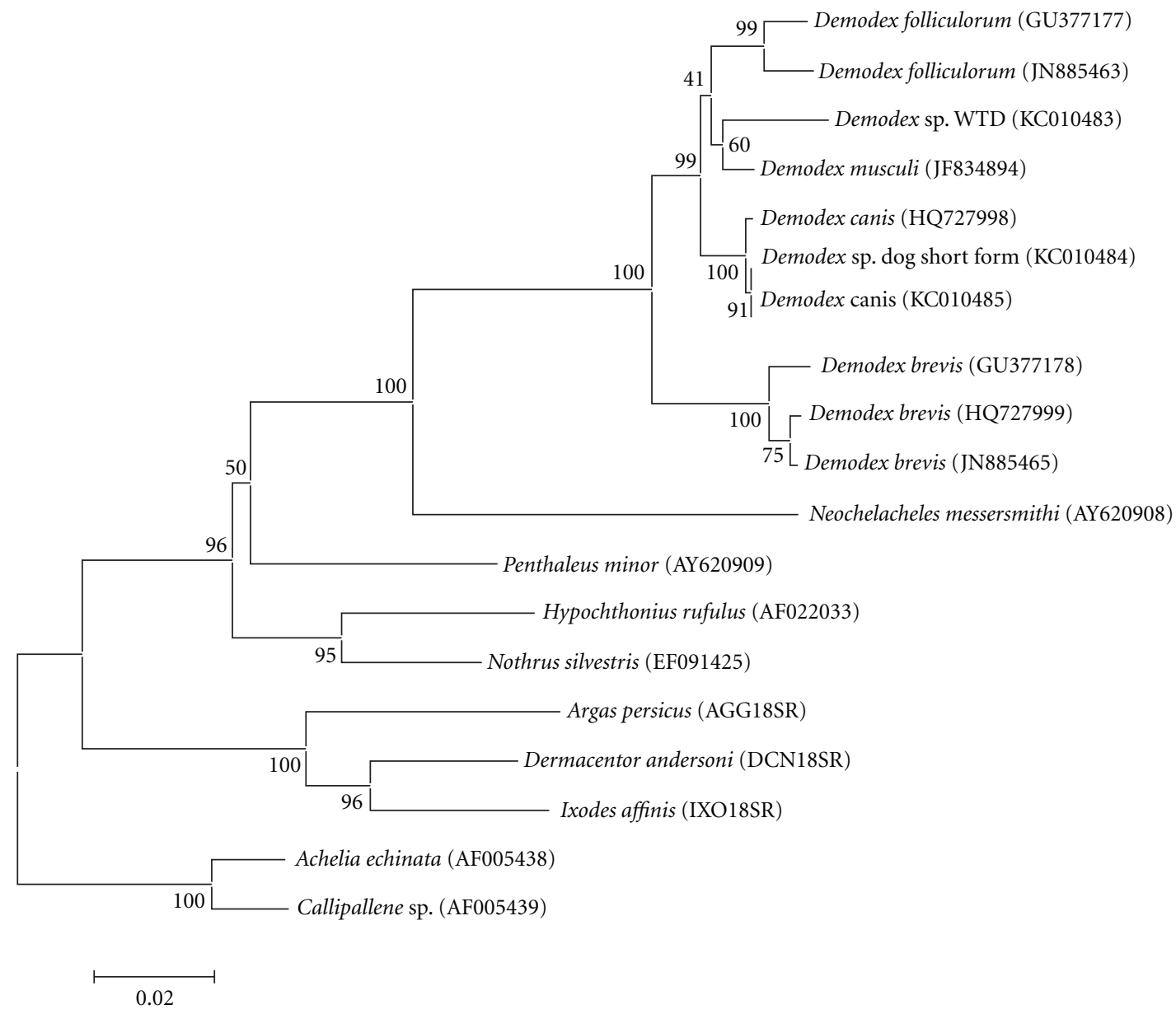

FIgURE 8: Phylogenetic relationships of Demodex species with related mites. The Demodex sp. of WTD (white-tailed deer) and the two Demodex spp. samples obtained from dogs in the current study are bolded. Demodex ursi from the black bear was excluded because the sequence was shorter than other sequences. 
with nodular demodicosis which tends to be rare in cases of D. odocoilei infestation [5].

Based on phylogenetic analyses, the Demodex sp. from the white-tailed deer was most similar to D. musculi from laboratory mice. Interestingly, Demodex from deer, dogs, mice, and humans ( $D$. folliculorum) formed a clade separate from the other human-infesting Demodex species (D. brevis) (current study and [11]). The monophyletic Demodex group was related to $N$. messersmithi, another member of the superfamily Chelyetoidea supporting the classical relationships of this superfamily of mites and other recent studies on the phylogenetics of the Chelyetoidea [15, 19]. Use of the $18 \mathrm{~S}$ rRNA gene was useful in distinguishing most species in the current study and one previous study on human and canine species [11]. However, based on partial $18 \mathrm{~S}$ rRNA gene sequence, we were unable to distinguish the mites from white-tailed deer and bear as different Demodex species; however, Demodex ursi from black bears [20] and the Demodex sp. from white-tailed deer are morphologically distinct. In the future, Demodex sequence data of more variable gene targets from additional species of Demodex obtained from a diverse set of hosts will allow studies on the diversity of Demodex sp. infecting different host species as well as studies on host specificity.

\section{Acknowledgments}

The authors thank N. Ruffin for the Demodex canis sample. S.E.C, and this research were primarily supported by the Merck-Merial Georgia Veterinary Scholars Program. Additional support came from the Cooperative Agreement 0696130032CA, Veterinary Services, APHIS, USDA; the Cooperative Agreement 06ERAG0005, Biological Resources Division, United States Geological Survey, USDI; and the Sponsorship of SCWDS by the fish and wildlife agencies of Alabama, Arkansas, Florida, Georgia, Kentucky, Kansas, Louisiana, Maryland, Mississippi, Missouri, North Carolina, Ohio, Puerto Rico, South Carolina, Tennessee, Virginia, and West Virginia. Support from the states to SCWDS was provided in part by the Federal Aid to Wildlife Restoration Act (50 Stat. 917).

\section{References}

[1] W. B. Nutting and C. E. Desch, "Demodex canis: redescription and reevaluation," The Cornell Veterinarian, vol. 68, no. 2, pp. 139-149, 1978.

[2] C. E. Desch and A. Hillier, "Demodex injai: a new species of hair follicle mite (Acari: Demodecidae) from the domestic dog (Canidae)," Journal of Medical Entomology, vol. 40, no. 2, pp. 146-149, 2003.

[3] V. Bukva, "Demodex kutzeri sp. n. (Acari: Demodicidae), an identical parasite of two species of deer, Cervus elaphus and C. nippon pseudaxis," Folia Parasitologica, vol. 34, no. 2, pp. 173-181, 1987.

[4] C. E. Desch and W. B. Nutting, "Demodex adocoilei sp. nov. from the white-tailed deer, Odocoileus virginianus," Canadian Journal of Zoology, vol. 52, no. 6, pp. 785-789, 1974.
[5] C. E. Desch, J. J. Andrews, L. A. Baeten et al., "New records of hair follicle mites (Demodecidae) from North American Cervidae," Journal of Wildlife Diseases, vol. 46, no. 2, pp. 585-590, 2010.

[6] H. F. Matthes, "Investigations of pathogenesis of cattle demodicosis: sites of predilection, habitat and dynamics of demodectic nodules," Veterinary Parasitology, vol. 53, no. 3-4, pp. 283-291, 1994.

[7] F. E. Kellogg, T. P. Kistner, R. K. Strickland, and R. R. Gerrish, "Arthropod parasites collected from white-tailed deer," Journal of Medical Entomology, vol. 8, no. 5, pp. 495-498, 1971.

[8] J. W. Carpenter, J. C. Freeny, and C. S. Patton, "Occurrence of Demodex Owen 1843 on a white-tailed deer from Oklahoma," Journal of Wildlife Diseases, vol. 8, no. 2, pp. 112-114, 1972.

[9] R. J. Bildfell, J. W. Mertins, J. A. Mortenson, and D. F. Cottam, "Hair-loss syndrome in black-tailed deer of the Pacific Northwest," Journal of Wildlife Diseases, vol. 40, no. 4, pp. 670-681, 2004.

[10] M. L. Gentes, H. Proctor, and G. Wobeser, "Demodicosis in a mule deer (Odocoileus hemionus hemionus) from Saskatchewan, Canada," Journal of Wildlife Diseases, vol. 43, no. 4, pp. 758-761, 2007.

[11] Y. E. Zhu and L. P. Wu, "Phylogenetic relationships in Demodex mites (Acari: Demodicidae) based on mitochondrial 16S rDNA partial sequences," Parasitology Research, vol. 111, no. 3, pp. 1113-1121, 2012.

[12] V. Bukva and J. Preisler, "Observations on the morphology of the hair follicle mites (Acari: Demodicidae) from Cervus elaphus L., 1758 including description of Demodex acutipes sp. n.", Folia Parasitologica, vol. 35, no. 1, pp. 67-75, 1988.

[13] E. Kutzer and W. Grünberg, "Demodicosis in red-deer (Cervus elaphus)," Zeitschrift für Parasitenkunde, vol. 40, no. 1, pp. 9-17, 1972.

[14] W. C. Black IIII, J. S. H. Klompen, and J. E. Keirans, "Phylogenetic relationships among tick subfamilies (Ixodida: Ixodidae: Argasidae) based on the 18S nuclear rDNA gene," Molecular Phylogenetics and Evolution, vol. 7, no. 1, pp. 129-144, 1997.

[15] A. V. Bochkov and B. M. OConnor, "Phylogeny, taxonomy and biology of mites of the genera Chelacheles and Neochelacheles (Acari: Cheyletidae)," Invertebrate Systematics, vol. 18, no. 5, pp. 547-592, 2004.

[16] S. Kumar, K. Tamura, and M. Nei, "MEGA: molecular evolutionary genetics analysis software for microcomputers," Computer Applications in the Biosciences, vol. 10, no. 2, pp. 189-191, 1994.

[17] (AFIP) Armed Forces Institute of Pathology, "Demodicosis in a white-tailed deer. Case No. IIRA415,” 1999.

[18] C. M. Jacques, J. A. Jenks, M. B. Hildreth, R. J. Schauer, and D. D. Johnson, "Demodicosis in a white-tailed deer in South Dakota," The Prairie Naturalist, vol. 33, pp. 221-226, 2001.

[19] J. C. Turner and J. Cano, "Demodectic mange in a whitetailed deer from Walker County, Texas," Journal of Medical Entomology, vol. 45, no. 3, pp. 572-575, 2008.

[20] Y. E. Zhao, L. P. Wu, L. Hu, Y. Xu, Z. H. Wang, and W. Y. Liu, "Sequencing for complete rDNA sequences (18S, ITS1, 5. 8S, ITS2, and 28S rDNA) of Demodex and phylogenetic analysis of Acari based on 18S and 28S rDNA," Parasitology Research, vol. 21, no. 5, pp. 2109-2114, 2012. 

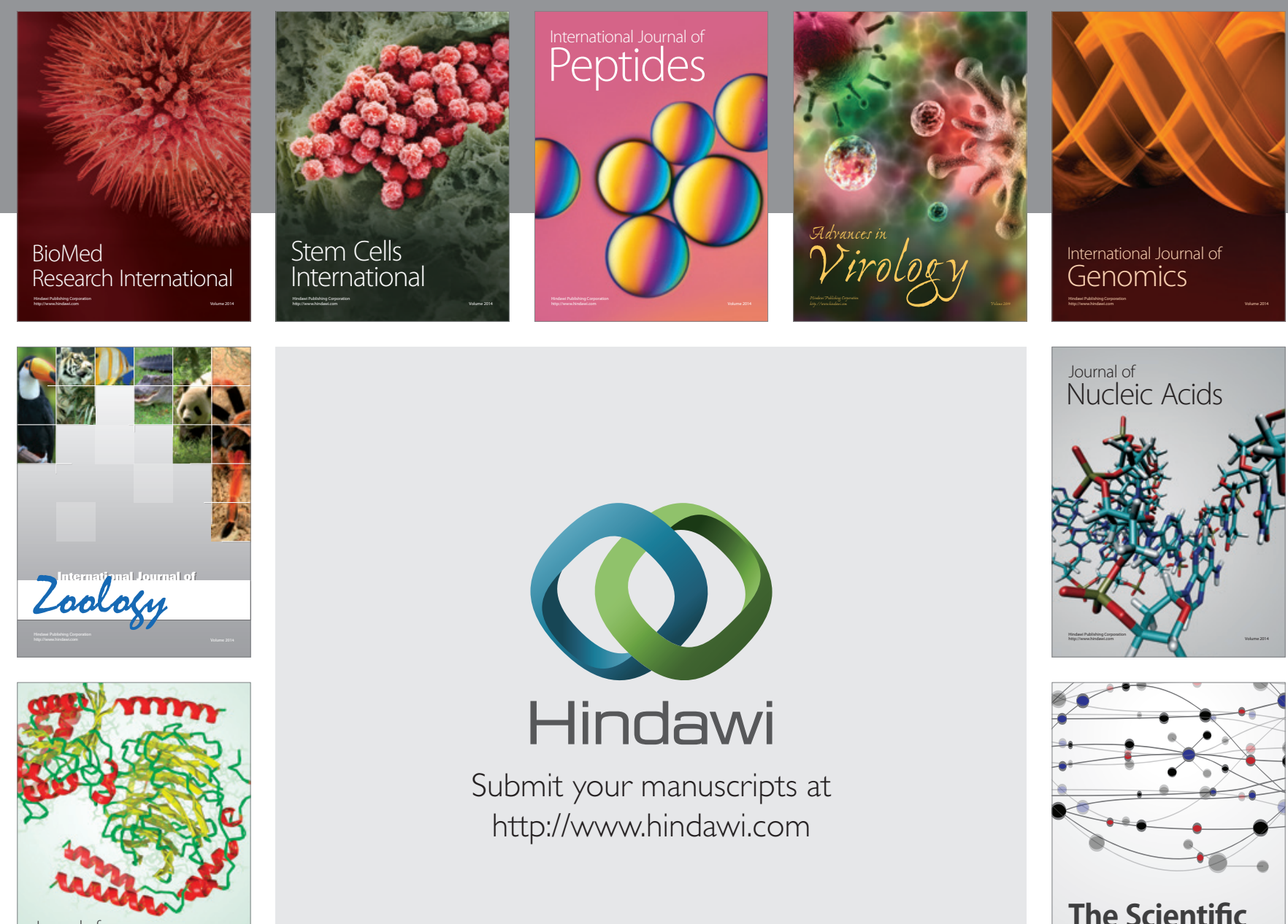

Submit your manuscripts at

http://www.hindawi.com

Journal of
Signal Transduction
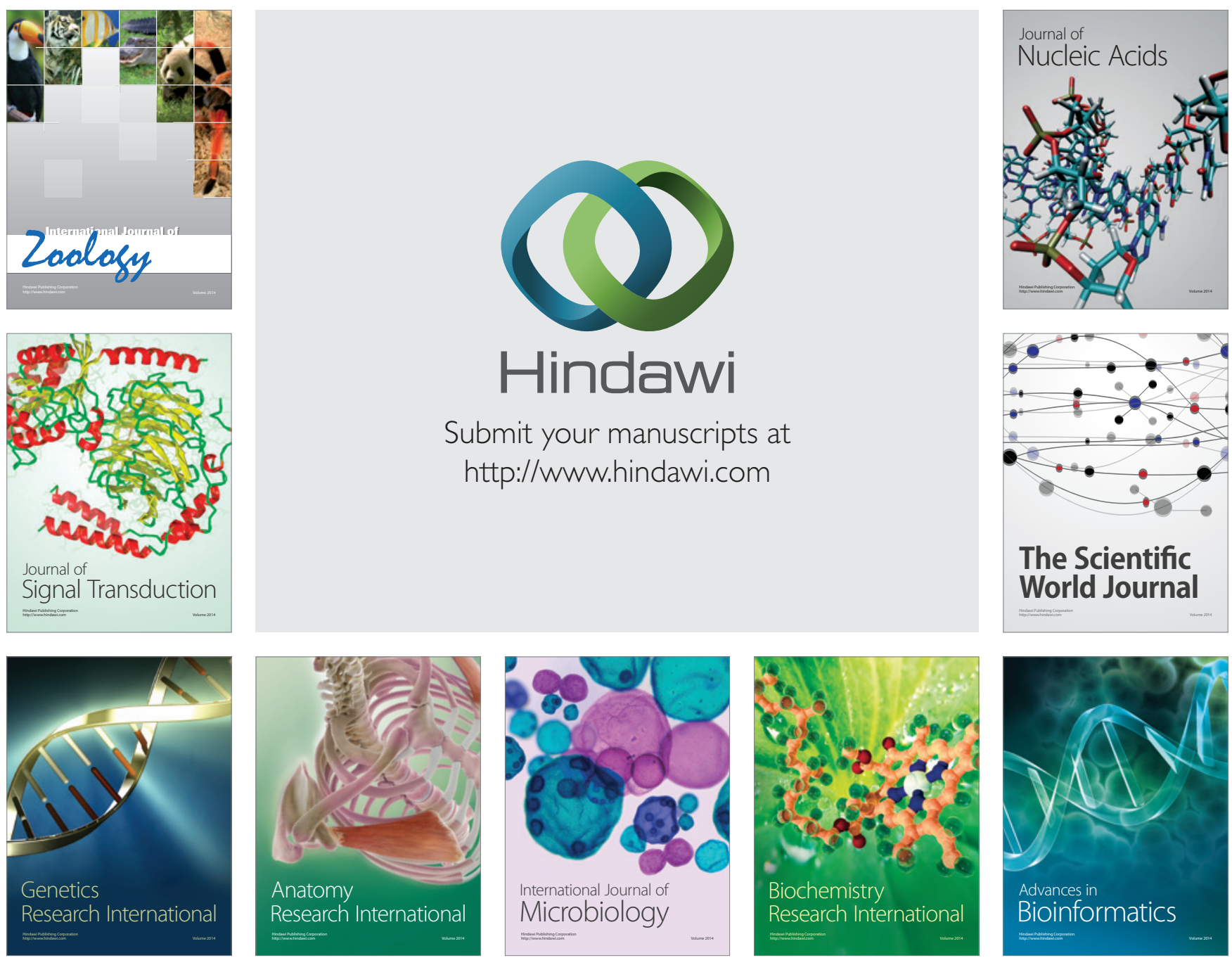

The Scientific World Journal
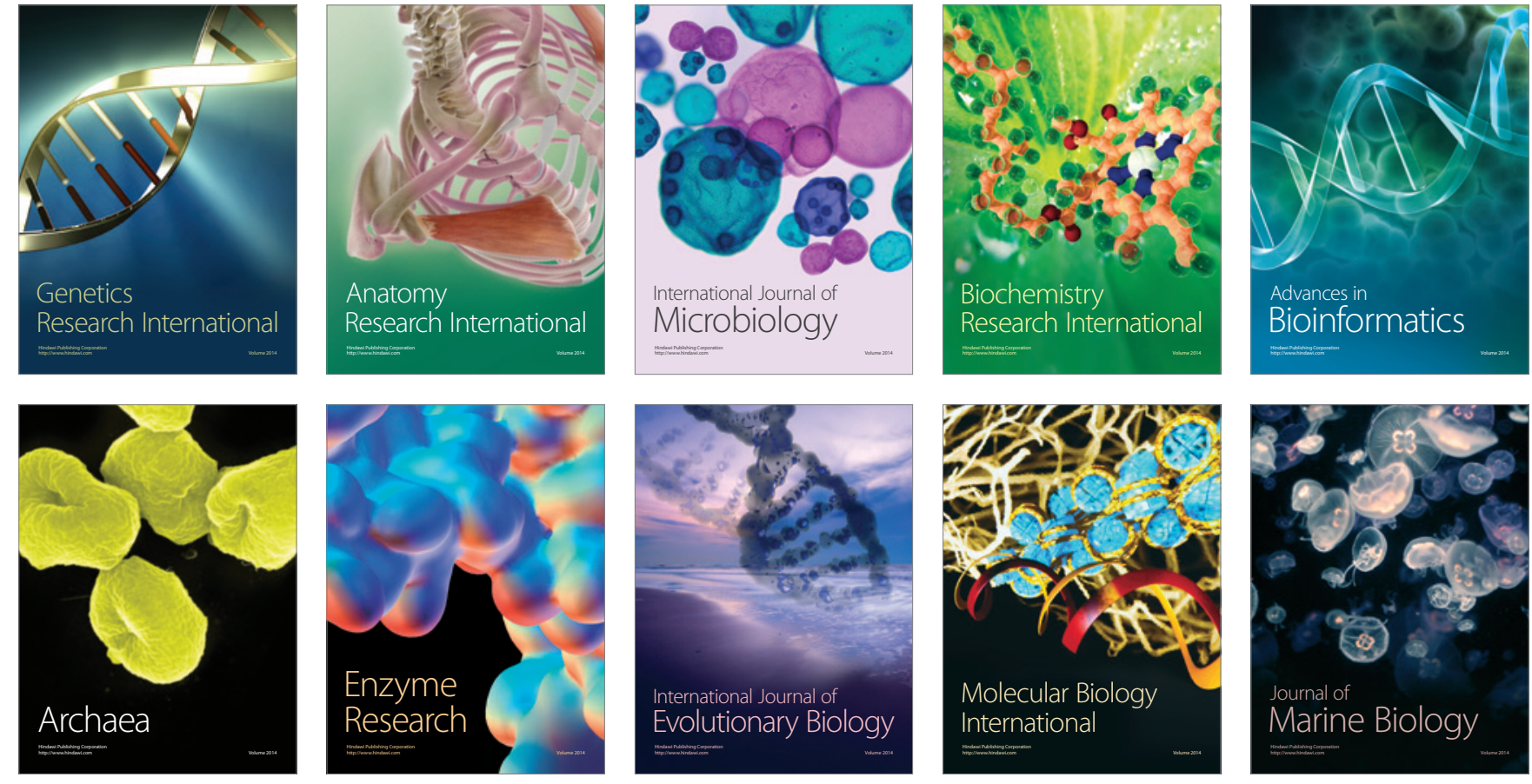